\title{
Evaluación de la resistencia adhesiva entre el poste de fibra de vidrio y el muñón de resina utilizando diferentes tratamientos de superficie
}

Tensile Bond strength evaluation between glass fiber post and resin core using different surface treatments.

\author{
Sayuri Dominguez ${ }^{1 \mathrm{1a}}$, Diana Castillo Andamayo ${ }^{1, \mathrm{a}, \mathrm{b}, \mathrm{c}}$, Oswaldo Ramos ${ }^{1, \mathrm{a}, \mathrm{b}, \mathrm{c}}$, Aníbal Rozas ${ }^{\text {2,d,e }}$
}

\section{RESUMEN}

La calidad de unión entre la superficie del poste de fibra de vidrio y el muñón es esencial para formar un solo núcleo. Si esta unión es afectada, ocasionaría una mala distribución de las fuerzas oclusales. Objetivos: Comparar la resistencia adhesiva entre el poste de fibra de vidrio y muñón de resina utilizando diferentes tratamientos de superficie. Material y métodos: La resistencia adhesiva se evaluó mediante el método de tracción. El diseño del estudio fue analítico, corte transversal y experimental in vitro.La población estuvo conformada por postes de fibra de vidrio estériles. El tamaño muestral fue de 24 postes; fueron divididos en cuatro grupos según el tratamiento de superficie: Grupo I, control negativo, con silano (60s); grupo II, control positivo, con peróxido de hidrógeno al 9\%(30 volúmenes, 10min) seguido de lavado y silano (60s); grupo III y IV, grupos experimentales, con ácido fosfórico al 37\%(15s) lavado y silano(60s) y con clorhexidina al 2\%(60s) lavado y silano (60s).Luego, se elaboró un muñón para cada poste, la muestra fue colocada en la máquina de ensayo universal. La fuerza de tracción fue recopilada en Mpa. Resultados: Se determinó que el grupo silano obtuvo una media de 15,38; peróxido de hidrógeno al 9\%+silano 20,28; ácido fosfórico al 37\%+silano 17,08; y clorhexidina al 2\%+silano 13,44 Según la prueba de Shapiro Wilk, los cuatro grupos presentan distribución normal $(p>0,05)$ y siguiendo la prueba de Barlett, se aprobó el supuesto de homogeneidad ( $p>0,05)$. Mediante la prueba multivariada de ANOVA; se determinó que existe diferencia estadística $(\mathrm{p}=0,0194)$, al menos, en un par de los cuatro grupos. Conclusiones: Existe diferencia estadísticamente significativa entre el grupo de peróxido de hidrógeno al 9\%(30 volúmenes, 10 $\mathrm{min})+$ silano $(60 \mathrm{~s})$ y clorhexidina al $2 \%(60 \mathrm{~s})+\operatorname{silano}(60 \mathrm{~s})(\mathrm{p}=0,025)$, siendo el primero, la sustancia con mayor resistencia adhesiva.

PALABRAS CLAVE: Clorhexidina, peróxido de hidrógeno, poste de fibra de vidrio.

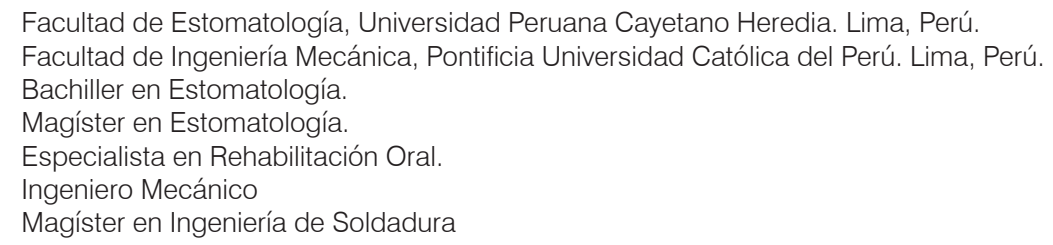




\section{SUMMARY}

The interface quality between the glass fiber post surface and core is essential to form a monoblock. If this interface is affected, a wrong distribution of occlusal forces could be caused. Objectives: To determine the bond strength between the glass fiber post and the resin core using different post surface treatments. Material and methods: The bond strength was evaluated by the traccional method. The study design was analytical, cross-sectional and experimental in vitro. The population were sterilized glass fiber posts. The sample size were 24 posts which were divided into four groups following the post surface treatment: Group I, negative control, with silane (60s); group II, positive control, with $9 \%$ hydrogen peroxide (30 volumes, $10 \mathrm{~min}$ ) followed by cleansing and silane (60s); groups III and IV, experimental groups, with phosphoric acid 37\%(15s) followed by cleansing and silane(60s) and with clorhexidine $2 \%(60 \mathrm{~s})$ followed by cleansing and silane(60s). Therefore, a resin core was devised for each post, the sample was placed in the universal traction machine. The tensile force was collected in Mpa. Results: It was determined that the silane group obtained a mean of $15.38,9 \%$ hydrogen peroxide+silane group 20.28, phosphoric acid 37\%+silane group 17.08, and Clorhexidine $2 \%+$ silane group 13.44. According to the Shapiro-Wilk test, all four groups presented normal distribution $(\mathrm{p}>0.05)$ and following Barlett's test, the homogeneity of variances assumption was approved ( $\mathrm{p}>0.05)$. Through the multifactor ANOVA test, it was determined that there is statistically significant difference $(\mathrm{p}=0.0194)$, at least in a couple of four groups. Conclusions: There is statistically significant difference between $9 \%$ hydrogen peroxide( 30 volumes, $10 \mathrm{~min})+-$ silane(60s) group and the Clorhexidine $2 \%(60 \mathrm{~s})+$ silane(60s) group $(\mathrm{p}=0,025)$. Being the former glass surface treatment, the highest bond strength.

\section{KEYWORDS: Chlorhexidine, hydrogen peroxide, glass fiber post.}

\section{INTRODUCCIÓN}

Los postes de fibra de vidrio son comúnmente utilizados hoy en día en el tratamiento posendodóntico de piezas dentarias con insuficiente estructura coronaria teniendo como alguno de sus objetivos formar un solo núcleo en conjunto con la pieza dentaria tratada. Por tanto, es importante la calidad de unión establecida entre la superficie del poste y el muñón (1-5).

Durante muchos años se ha investigado los procedimientos del tratamiento de superficie en postes de fibra de vidrio los cuales son mecánicos, químicos o la combinación de ambos, encontrando en los primeros la aplicación del peróxido de hidrógeno, silano, ácido fosfórico, entre otros $(6,7)$.

Existen diversos factores que puedan afectar la unión del poste al muñón, ocasionando diversas complicaciones con respecto a la distribución de fuerzas oclusales; por lo cual, es importante la elección de un tratamiento de superficie adecuado y lograr éxito en el tratamiento protésico $(3,8)$.

El propósito de la presente investigación fue comparar la resistencia adhesiva entre el poste de fibra de vidrio y el muñón de resina utilizando diferentes tratamientos de superficies sobre los postes.

\section{MATERIAL Y MÉTODOS}

El presente estudio de tipo analítico, experimental in vitro y corte transversal tuvo como tamaño muestral seis postes de fibra de vidrio libres de contaminación y estériles por cada grupo de estudio siendo un total de 24 especímenes; este se determinó teniendo como referencia los datos obtenidos de la prueba piloto realizada previa al experimento, donde se aplicó la fórmula de comparación de medias teniendo como resultado 5,50. El estudio se llevó a cabo en el Laboratorio de Materiales, sección Ingeniería Mecánica, de la Pontificia Universidad Católica del Perú (PUCP). Previa autorización del Comité de Investigación de la Facultad de Estomatología de la Universidad Peruana Cayetano Heredia y el Comité Institucional de Ética (CIE) del mismo. Siendo el código de aprobación CAREG-ORVEI-006-016, SIDISI 66386.

Para el estudio se tomaron como criterios de inclusión los postes de fibra de vidrio radiopacos pertenecientes a la misma empresa; con igual longitud, diámetro. Además del material de reconstrucción de muñones 
de polimerización dual. Se consideraron como criterios de exclusión los postes de diferentes empresas, longitud o diámetro, y los postes con algún defecto en la superficie.

Previo a la elaboración de la muestra, se confeccionó un aditamento de plástico que permitió realizar cuatro muestras a la vez que mantuvieron los postes en el centro de cada molde. Además, se confeccionó cuatro moldes de plástico cilíndrico con un diámetro de 6 $\mathrm{mm}$ y una altura de $10 \mathrm{~mm}$; y cuatro moldes de plástico cilíndrico con un diámetro de $6 \mathrm{~mm}$ y una altura de $4 \mathrm{~mm}$. Se utilizaron 24 postes de fibra de vidrio White Post DC 0,5 (FGM, Joinville, SC-Brasil) de diámetro $1,4 \mathrm{~mm}$ y longitud de $19 \mathrm{~mm}$ esterilizados a $121^{\circ} \mathrm{C}$ y 15 psi, según las recomendaciones del fabricante. Todos los postes fueron divididos en cuatro grupos, al azar, de acuerdo a los diferentes tratamientos de superficie:

- Grupo I - Grupo control negativo, con aplicación de silano Bis-SilaneTM 2 Part-Porcelain Primer (Bisco Inc., Schaumburg, IL, U.S.A.) por 60 segundos. - Grupo II - Grupo control positivo con aplicación de peróxido de hidrógeno al 9\%-30 volúmenes (Farmacia Universal, Lima, Perú) en la superficie del poste por 10 minutos, seguido del lavado con agua desionizada y aplicación de una capa de silano por 60 segun$\operatorname{dos}(9,10,11)$.

- Grupo III - Grupo experimental con ácido fosfórico al 37\% ETCH-37TM (Bisco Inc., Schaumburg, IL, U.S.A.) fue aplicado en la superficie del poste por 15 segundos, lavado con agua desionizada y aplicación de una capa de silano por 60 segundos (5).

- Grupo IV - Grupo experimental con solución de digluconato de Clorhexidina 2\% (FGM, Joinville, SC-Brasil) fue aplicado en la superficie del poste por 60 segundos, lavado con agua desionizada y aplicación de una capa de silano por 60 segundos.

Luego se procedió a la elaboración de la muestra, para lo cual se realizó la elaboración del soporte de la siguiente manera: cuatro moldes previamente envaselinados de $10 \mathrm{~mm}$ de altura fueron colocados en el aditamento de plástico, éste fue colocado en una platina estéril; luego se inyectó la resina de polimerización dual LuxaCore Z Dual, A3 (DMG, Hamburgo-Alemania) en cada molde utilizando la boquilla automix. Cada poste, utilizando la parte cónica del mismo, fue colocado de manera perpendicular en el centro del molde, luego se procedió a polimerizar por 50 segundos con luz halógenaLITEXTM 680A (Dentamerica Inc., California, U.S.A.) con intensidad luminosa de $526 \mathrm{~mW} / \mathrm{cm}^{2}$ (esta fue calibrada por el radiómetro DigiRate Radiometer LM-100 LED/Halogen Light Energy \& Temperature (Monitex Industrial CO., New Taipei, Taiwan)), directamente desde el orificio del molde lo más cercano posible al material siguiendo las indicaciones del fabricante, quien indica que se debe usar como mínimo una intensidad luminosa de $400 \mathrm{~mW} / \mathrm{cm}^{2}$ y el proceso de fotocurado es de 20 s $($ capa $\leq 2 \mathrm{~mm})$ y 40 s (capa $\leq 4 \mathrm{~mm})$. Por último, la parte cónica de la muestra que estuvo en contacto con la platina fue polimerizado por 50 segundos adicionales (figura 1 ).

Después de elaborar el soporte de todos los postes se procedió con el muñón, para lo cual se realizó de la siguiente manera: cuatro moldes previamente envaselinados, esta vez de $4 \mathrm{~mm}$ de altura fueron colocados en el aditamento de plástico, éste fue colocado en una platina estéril; luego, se inyectó la resina de polimerización dual en cada molde utilizando la boquilla automix. Los postes con soporte previamente confeccionados fueron utilizados, colocando esta vez la parte cilíndrica del poste de manera perpendicular en el centro del molde, luego se procedió a polimerizar por 40 segundos con luz halógena directamente desde el orificio del molde lo más cercano posible al material siguiendo las indicaciones del fabricante (figura 2 y figura3).

Una vez obtenida las muestras, se procedió con el ensayo de tracción para lo cual se confeccionaron dos aditamentos que ayudaron a la colocación de cada muestra en la máquina de ensayo universal modelo Z2.5 BT1-FR0.5TS.D14 (Zwick/Roell, UlmAlemania). Las muestras no fueron almacenadas bajo ninguna condición, debido a que en el presente estudio no se buscó resultados después de un proceso de envejecimiento, además según Sahafi et al., (12) y Bitter et al., (13) la resistencia adhesiva entre el poste y el muñón depende más del material del poste y el tratamiento de superficie que de la duración y condiciones de almacenamiento. Cada muestra fue colocada en la máquina de ensayo universal, produciendo una fuerza, a una velocidad constante de $3 \mathrm{~mm} / \mathrm{min}$ hasta que ocurra la fractura entre el poste de fibra de vidrio y el muñón de resina (figura 4 y figura 5). El esfuerzo de tracción de cada muestra fue 


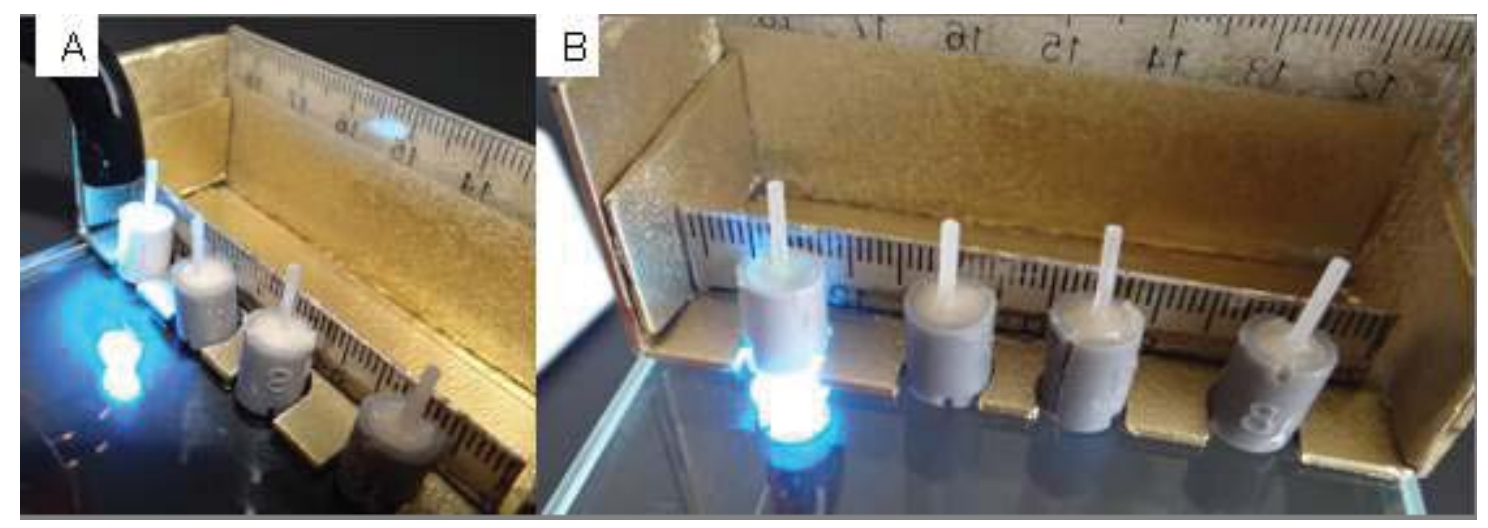

Figura 1. A y B. Polimerización del soporte por 50 segundos en cada lado.

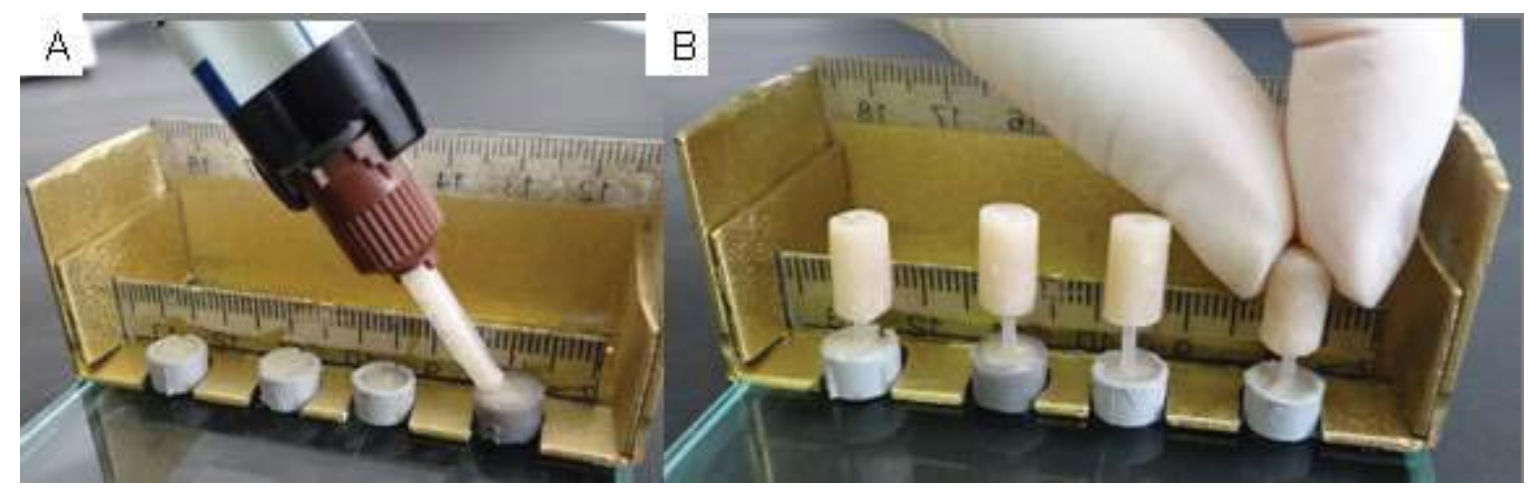

Figura 2. A y B: Elaboración del muñón de resina (parte cilíndrica)

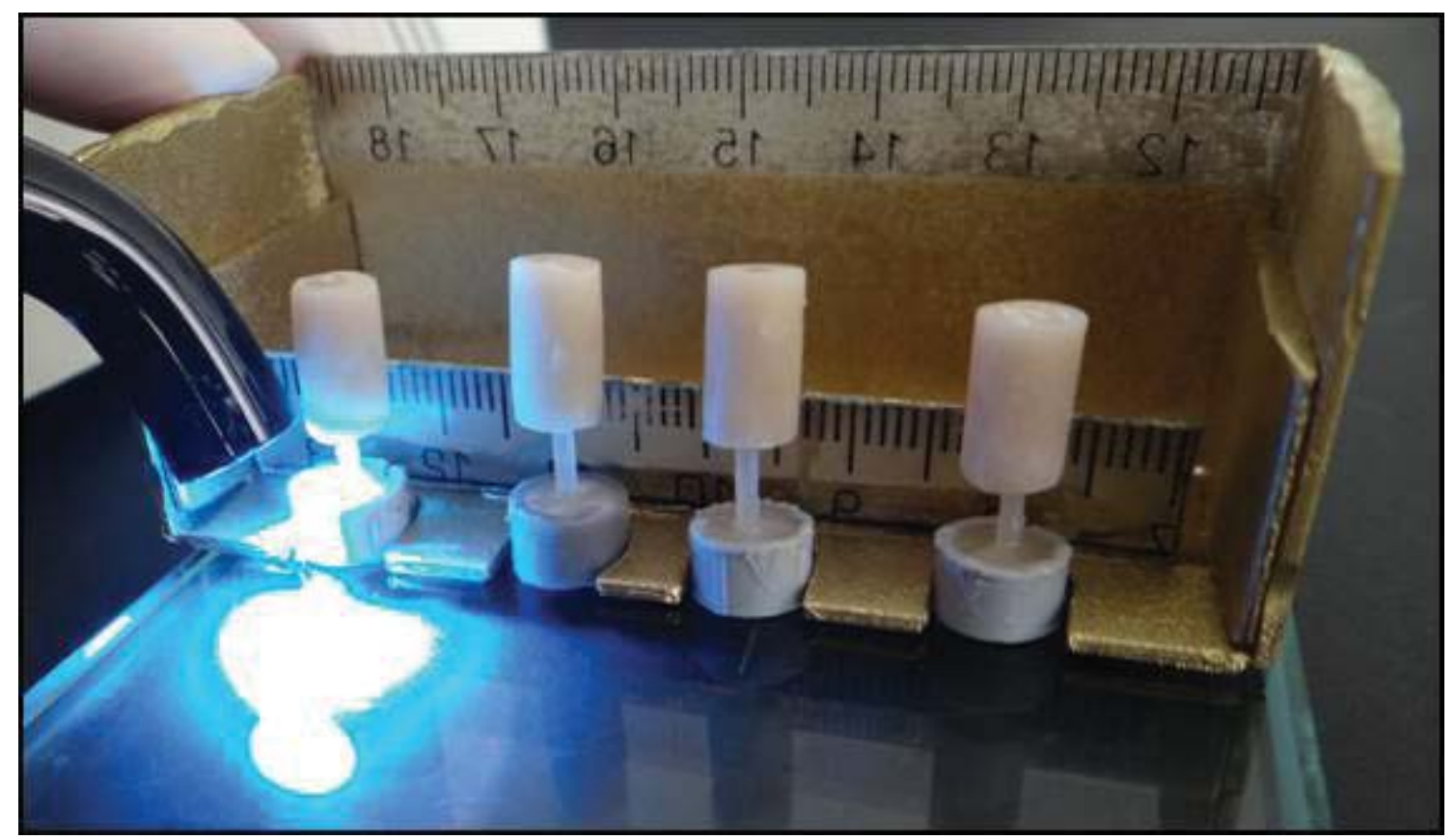

Figura 3. Polimerización del muñón de resina por 40 segundos. 


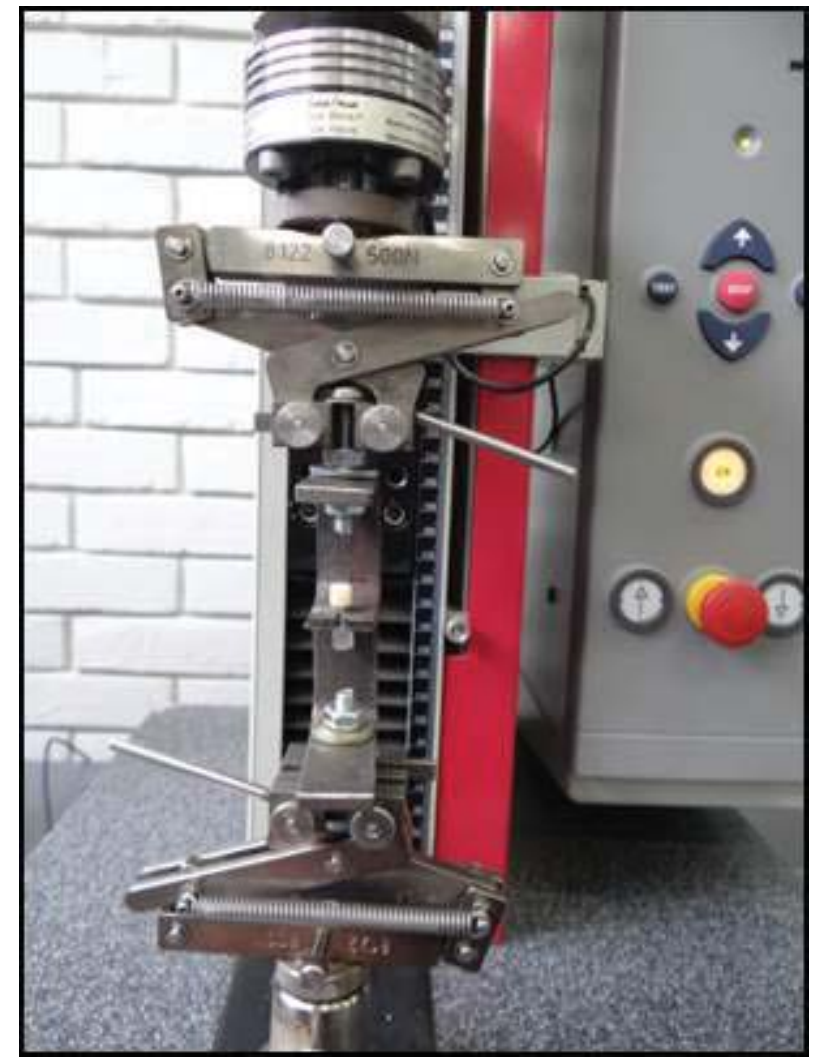

Figura 4. Máquina de ensayo universal modelo Z2.5 BT1-FR0.5TS.D14 (Zwick/Roell, Ulm-Alemania).

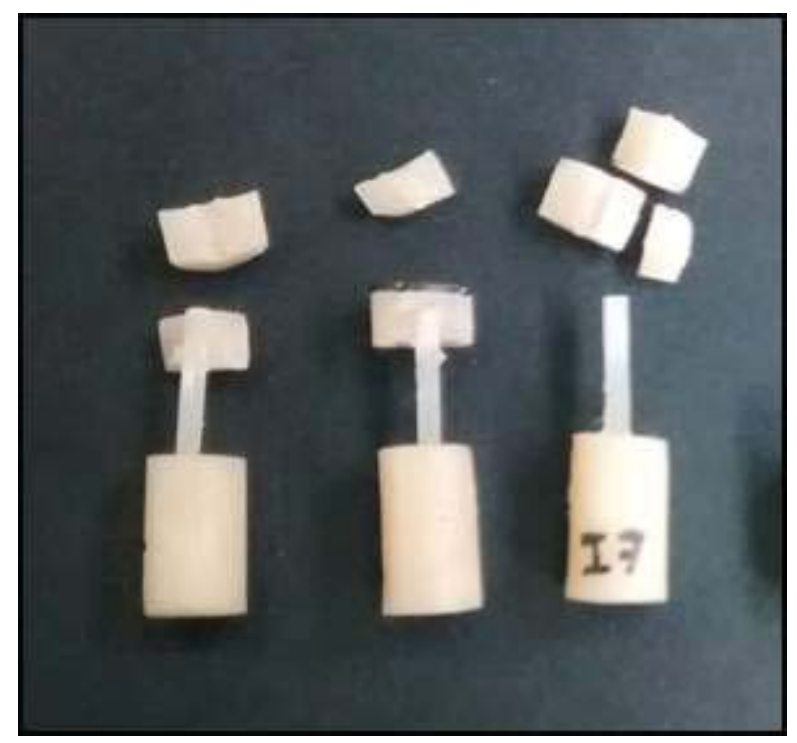

Figura 5. Ensayo de tracción hasta la fractura 
calculado de la siguiente manera: fuerza a la fractura $(\mathrm{N}) /$ área de superficie $\left(\mathrm{mm}^{2}\right)$ expresado en Mpa; éstos datos fueron recopilados en una ficha de recolección de datos y analizados posteriormente utilizando el software STATA versión 12.

Para el análisis univariado, las variables cuantitativas se realizaron con las medidas de tendencia central (media y mediana), y las medidas de dispersión (desviación estándar, intercuartílica y varianza). Respecto al análisis bivariado, debido a que la distribución fue normal se utilizó la prueba $t$ student para muestras independientes. Además, se utilizó la prueba multivariada de ANOVA de un factor y la prueba Pos Hoc de Scheffe también conocida como comparaciones múltiples.

\section{RESULTADOS}

La presente investigación in vitro tuvo como propósito comparar la resistencia adhesiva entre el poste de fibra de vidrio y el muñón de resina, luego de utilizar diferentes tratamientos de superficies sobre los postes. Resultados, párrafo 1: Los tratamientos de superficie que se utilizaron fueron: Grupo control: Silano (60s); peróxido de hidrógeno al 9\% (30 volúmenes, $10 \mathrm{~min})+$ silano (60s), negativo y positivo respectivamente.

Los resultados de las medidas de tendencia central (media y mediana) y los valores de la resistencia a la tracción de todos los grupos fueron calculados de la siguiente manera, el grupo silano obtuvo una media de $15,38 \pm 4,72$; el peróxido de hidrógeno $9 \%$ y silano, $20,28 \pm 3,10$; ácido fosfórico $37 \%$ y silano, $17,08 \pm 3,29$; y la clorhexidina $2 \%$ y silano, $13,44 \pm 2,41$. Siguiendo éstas medidas, el grupo de peróxido de hidrógeno 9\% y silano; y la clorhexidina $2 \%$ y silano presentan la mayor y menor resistencia adhesiva respectivamente en comparación con las demás sustancias evaluadas. Además, se pudo observar que la dispersión (desviación estándar y dispersión intercuartílica) de los datos es baja $(\mathrm{CV}<0,33)$, por lo que se decidió elegir la media aritmética y desviación estándar para la realización de los gráficos (tabla 1 y gráfico 1 ).

Para elegir si se realiza una prueba paramétrica o no paramétrica es necesario comprobar si los valores presentan distribución normal y varianza homogénea. La prueba de Shapiro Wilk nos muestra que los cuatro grupos presentan distribución normal $(\mathrm{p}>0,05)$ y la prueba de Barlett nos muestra que se aprueba el supuesto de homogeneidad ( $p>0,05)$. Esta prueba nos permitió decidir que las pruebas paramétricas son las de mejor opción. Por tanto, se elige utilizar la prueba multivariada de ANOVA de un factor (tabla 2).

Al evaluar la resistencia adhesiva de los diferentes tratamientos de superficie entre el poste de fibra de vidrio y el muñón de resina, mediante la prueba multivariada de ANOVA de un factor; se concluye que existen diferencias estadísticamente significativas $(\mathrm{p}=0,0194)$, al menos, en un par de los cuatro grupos (tabla 3).

Cuando se hizo la comparación de la resistencia ad-

Tabla 1. Medidas de tendencia central y dispersión (MPa) según tipo de material.

\begin{tabular}{lccccc}
\hline Material & Media & D.E. & Mediana & DIC & cv \\
\hline Silano & 15,388 & 4,728 & 15,656 & 2,665 & 0,307 \\
$\mathrm{H}_{2} \mathrm{O}_{2} 9 \%+$ Silano & 20,283 & 3,103 & 20,119 & 2,615 & 0,153 \\
$\mathrm{H}_{3} \mathrm{PO}_{4} 37 \%+$ Silano & 17,080 & 3,291 & 17,683 & 2,493 & 0,193 \\
$\mathrm{CHX} 2 \%+$ Silano & 13,444 & 2,413 & 14,179 & 1,999 & 0,179 \\
Total & 16,549 & 4,142 & 16,455 & 2,442 & 0,249 \\
\hline
\end{tabular}




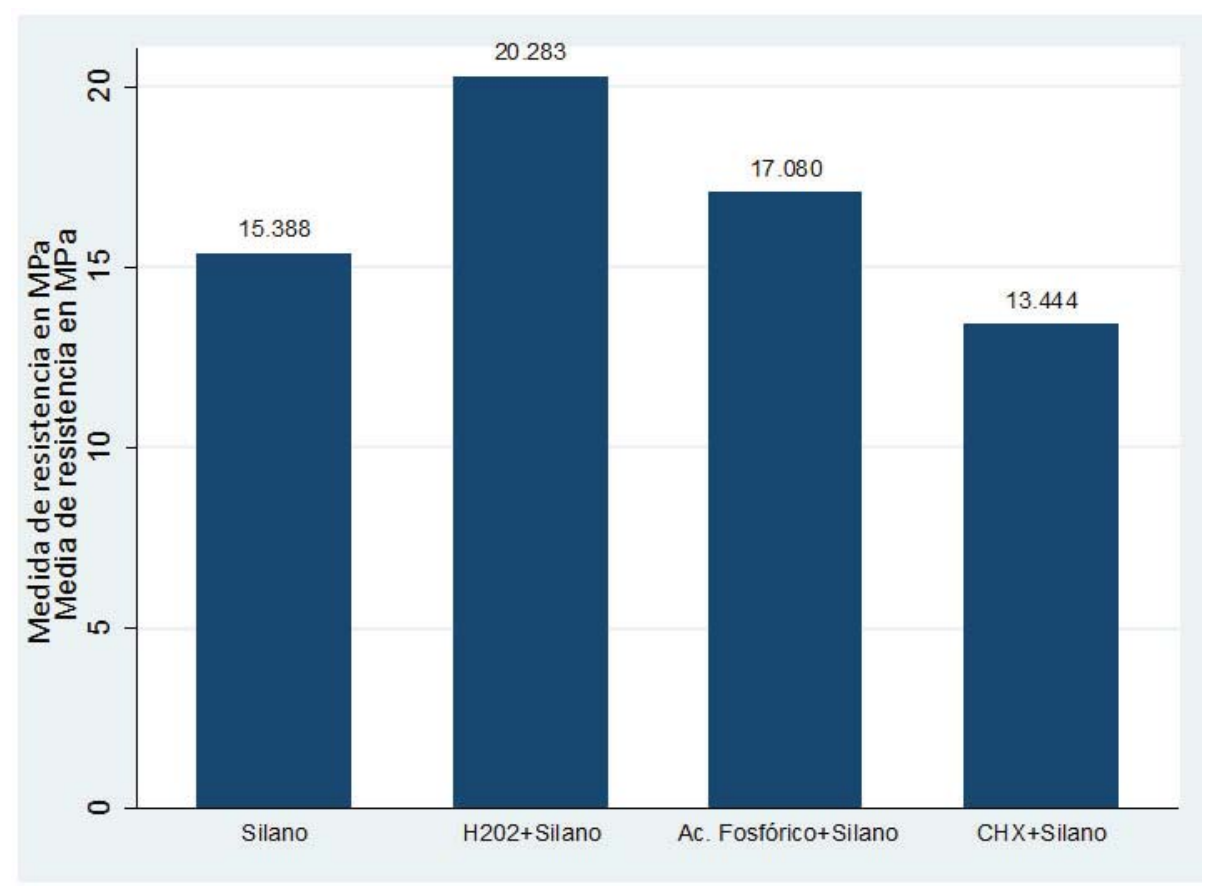

Gráfico 1. Media aritmética según tipo de material

Tabla 2. Supuesto de Normalidad e Igualdad de Varianzas

\begin{tabular}{clccccc}
\hline Grupos & Material & Obs & skewness & kurtosis & Z & Prob $>\mathbf{z}$ \\
\hline I & Silano & 6 & 0,122 & 2,285 & $-2,194$ & 0,98588 \\
II & $\mathrm{H}_{2} \mathrm{O}_{2} 9 \%+$ Silano & 6 & 0,209 & 1,484 & 0,271 & 0,39328 \\
III & $\mathrm{H}_{3} \mathrm{PO}_{4} 37 \%+$ Silano & 6 & $-0,067$ & 1,699 & $-0,604$ & 0,72722 \\
IV & CHX2\%+Silano & 6 & $-0,554$ & 1,809 & 0,149 & 0,44074 \\
\hline
\end{tabular}


Tabla 3. Análisis de Varianzas ANOVA de un factor para muestras independientes.

\begin{tabular}{|c|c|c|c|c|c|}
\hline Fuente & Grados Libertad & Suma de Cuadrados & $\begin{array}{c}\text { Media } \\
\text { Cuadrados }\end{array}$ & Distribución F & Valor $\mathbf{p}$ \\
\hline Entre grupos & 3 & 151,282 & 50,428 & 4,15 & 0,0194 \\
\hline Dentro de grupos & 20 & 243,223 & 12,161 & & \\
\hline Total & 23 & 394,507 & 17,152 & & \\
\hline
\end{tabular}

Tabla 4. Comparaciones múltiples.

\begin{tabular}{lccc}
\hline Materiales & Diferencias de medias (valor p) & \\
& Silano & $\mathbf{H}_{2} \mathbf{O}_{2}+$ Silano & Ac. Fosfórico+ Silano \\
$\mathrm{H}_{2} \mathrm{O}_{2} 9 \%+$ Silano & $4,89496(0,147)$ & & \\
$\mathrm{H}_{3} \mathrm{PO}_{4} 37 \%+$ Silano & $1,69151(0,871)$ & $-3,20345(0,486)$ & $-3,63567(0,377)$ \\
$\mathrm{CHX} 2 \%+$ Silano & $-1,94416(0,817)$ & $-6,83912(0,025)$ & - \\
\hline
\end{tabular}

hesiva de los diferentes tipos de sustancias usados en el tratamiento de superficie del poste de fibra de vidrio, mediante la prueba Pos Hoc de Scheffe también conocida como comparaciones múltiples; se pudo observar las diferencias estadísticas que se presentaban entre los grupos. Llegando a la conclusión que el grupo de peróxido de hidrógeno $9 \%$ y silano es el que presenta mayor resistencia, estas diferencias son estadísticamente significativas sólo con el grupo de clorhexidina $2 \%$ y silano $(\mathrm{p}=0,025)$; mientras que, con los otros grupos estas diferencias no son estadísticas significativas (tabla 4).

\section{DISCUSIÓN}

El presente estudio de tipo experimental in vitro tuvo como propósito comparar la resistencia adhesiva entre el poste de fibra de vidrio y el muñón de resina luego de la acción con diferentes tratamientos de superficie. Para este fin, se seleccionaron los siguientes tratamientos de superficie: Grupo control: Silano (60s); peróxido de hidrógeno al 9\% (30 volúmenes, $10 \mathrm{~min})+$ silano (60s), negativo y positivo respectivamente. Grupo experimental: ácido fosfórico 37\% (15s) y silano (60s), clorhexidina 2\% (60s) y silano (60s).

El tratamiento de la superficie del poste de fibra sirve para mejorar las propiedades de adhesión de un ma- terial, proporcionando una retención química y micromecánica. La posibilidad de combinar la retención química y micromecánica brindan aparentemente el mejor mecanismo de adhesión $(9,14)$.Debido a esto, muchos estudios han realizado la combinación de diversos componentes químicos y creando microrrugosidades en la superficie del poste de fibra de vidrio con el fin de obtener una mayor retención del poste de fibra al muñón de resina garantizando la longevidad de la prótesis (15).

Los postes de fibra están compuestos con finas fibras pretensadas colocadas de manera unidireccional, estas fibras pueden ser de carbono, vidrio o cuarzo. En su mayoría conglomeradas con una resina tipo epoxi, en algunos casos le añaden resinas Bis-GMA, en otros son a base de dimetacrilatos (10). Debido a esto, el peróxido de hidrógeno es usado con mucha frecuencia ya que expone la superficie de las fibras de vidrio disolviendo la matriz de resina epoxi a través del mecanismo de oxidación del sustrato, mejorando de esta manera la adhesión del poste de fibra de vidrio al muñón de resina $(1,2,4,16)$. El peróxido de hidrógeno brinda la retención micromecánica, y el silano la retención química mejorando la adhesión entre matrices inorgánicas y orgánicas gracias a su capacidad de reacción intrínseca dual logrando la unión del núcleo de matriz de resina y la fibra de vidrio expuesta. Sin 
embargo, esta unión interfacial no es elevada debido a la ausencia de la unión química entre los composites de resina basados en metacrilato y la matriz de resina epoxi de los postes de fibra de vidrio $(1-4,16)$. Por tanto, se sigue en la búsqueda de un tratamiento de superficie del poste de fibra de vidrio que supere al peróxido de hidrógeno junto con el silano.

Saker et al., (6), evaluaron el efecto del tratamiento de superficie de 40 postes reforzados con fibra de vidrio; donde utilizaron como tratamientos de superficie: arenado con óxido de aluminio (110um, 5s, 2,8 bar, $1 \mathrm{~cm}$ distancia), ácido fosfórico en gel al $37 \%$ (60s, 2 min en agua dionizada), peróxido de hidrógeno $24 \%$ (10min). Después del tratamiento todos los postes fueron sumergidos en agua por 30s y secados con aire para ser subdivididos en cinco especímenes los cuales recibirían tratamiento adhesivo con: silano (60s) y sistema adhesivo de un solo paso. Luego se aplicó composite de resina (Multicore) continuando con la prueba de push-out. Obteniendo la mayor fuerza de adhesión con el tratamiento de peróxido de hidrógeno y silano, seguido del grupo de arenado; el de menor fuerza fue en el grupo del ácido fosfórico con ambos agentes. En comparación con el presente estudio también se observa una mayor resistencia con el peróxido de hidrógeno y silano; diferenciándose en el tipo de prueba utilizada y en el porcentaje de peróxido de hidrógeno aplicado en los postes de fibra de vidrio.

Sharma et al., realizaron un estudio comparativo del efecto de diferentes sustancias en 30 postes de fibra de vidrio en la fuerza de adhesión con el material del muñón (4). Los tratamientos de la superficie que utilizaron fueron: silano $(60 \mathrm{~s})$, peróxido de hidrógeno $6 \%$ (20min), ácido fosfórico 37\% (20s). Los resultados demostraron que el peróxido de hidrógeno tuvo un gran impacto en el poste seguido del ácido fosfórico y silano. A diferencia del presente estudio, el porcentaje del peróxido de hidrógeno fue menor y no aplicaron una capa de silano. Sin embargo, observamos que también hubo una mayor resistencia con el peróxido de hidrógeno.

El presente estudio concuerda con el análisis crítico de las superficies de tratamiento en postes de fibra realizado por Machado et al., quienes se basaron en la base de datos de PubMed/Medline, consideraron los estudios con postes de fibra de cuarzo y de vidrio en la revisión. Se incluyeron 190 artículos, donde la mayoría de artículos se menciona que el uso del arenado afecta las propiedades físicas y mecánicas. Sin embargo, el uso del peróxido de hidrógeno con silano es el más prometedor ya que incrementa la fuerza de adhesión sin dañar los postes de fibra (7).

En referencia a los postes de fibra de cuarzo Shori et al., (3) y Sumitha et al., (2) obtuvieron resultados diferentes. Siendo Shori et al., (3) quien llegó a la conclusión que el peróxido de hidrógeno al 10\% junto con el silano logran una mayor unión del poste muñón comparado con primer y ácido fosfórico $37 \%$ y silano (3). Sumitha et al., logró un resultado significante utilizando permanganato de potasio como tratamiento de superficie en postes de fibra de cuarzo, en comparación con peróxido de hidrógeno $10 \%$ (10min), ácido fosfórico 37\% (5min) y silano (60s) (2). Este último estudio coincide con los resultados de Mazzitelli et al., quienes realizaron un estudio en postes de fibra de cuarzo, en el cual estos fueron acondicionados con diferentes químicos (ácido hidrofluorhídrico, permanganato de potasio, etóxido de sodio) con arenado (17). Llegando a la conclusión que el ácido hidrofluorhídrico daña la superficie de poste de cuarzo, mientras que el permanganato de potasio y el etóxido de sodio remueven parte de la matriz de resina epoxi incrementando la superficie de adhesión.

Con el presente estudio se determinó que el grupo de peróxido de hidrógeno $9 \%$ y silano presenta mayor resistencia, seguido de ácido fosfórico $37 \%$ y silano, silano solamente y la clorhexidina al $2 \%$ y silano presenta la menor resistencia de todas las sustancias evaluadas. Los hallazgos de este estudio "in vitro" permiten confirmar que el mejor tratamiento en postes de fibra de vidrio es el peróxido de hidrógeno $9 \%$ y silano.

Entre las sustancias más utilizadas para el tratamiento de los postes encontramos al peróxido de hidrógeno, el cual ha sido comparado con diversas sustancias a excepción de la clorhexidina; este último en la literatura actual hace referencia de ser un agente que mejora la adhesión con la resina. Recientemente la solución de clorhexidina ha sido considerada como potencial inhibidor de degradación de las fibras colágenas en la capa híbrida dentinaria favoreciendo la disminución de la degradación de la unión dentina-resina, es decir, una adhesión de mayor longevidad por la desactivación de las Metaloproteinazas (MMP). Además, esta solución es convencionalmente usada como irrigador dentinario por su propiedad antimicrobiana $(18,19)$. 
Por tanto se le atribuye al presente estudio la importancia práctica-clínica ya que ayudará al profesional a contar con la información necesaria para saber discriminar el uso de diversas sustancias en el acondicionamiento del poste de fibra de vidrio, facilitando así la mejor elección de la misma (aquel que le brinde mayor resistencia adhesiva) en su práctica odontológica. Se recomienda realizar mayores estudios de resistencia adhesiva con el tratamiento de superficie de solución de clorhexidina en postes de fibra de vidrio con diferentes porcentajes y tiempos, ya que es una sustancia con potencial adhesivo.

De acuerdo a la metodología utilizada en el presente estudio y a los resultados obtenidos, se puede concluir que sólo existe diferencia estadísticamente significativa entre el grupo de peróxido de hidrógeno al 9\%(30 volúmenes, $10 \mathrm{~min})$ y silano(60s)vs clorhexidina al $2 \%(60 \mathrm{~s})$ y silano(60s). Siendo las sustancias con mayor y menor resistencia adhesiva respectivamente.

\section{Agradecimiento:}

Se agradece la colaboración en el presente estudio del Dr. Enrique Pozo Merino, Ing. Daniel Calero Zavaleta y del Ing. Carlos Juárez Aparcana.

\section{Correspondencia:}

Sayuri Dominguez Cárdenas

Correo electrónico: sayuri.dominguez.c@upch.pe

\section{REFERENCIAS BIBLIOGRÁFICAS}

1. Miranda A, Resende V, De Sousa M, Guimarães M, Estrela C, Soares C. Effect of surface treatment of fiber glass post on bond strength to root dentin. Braz Dent J. 2014; 25(4): 314-20.

2. Sumitha M, Kothandaraman R, Sekar M. Evaluation of post-surface conditioning to improve interfacial adhesion in post-core restorations. J Conserv Dent. 2011; 14(1): 28-31.

3. Shori D, Pandey S, Kubde R, Rathod Y, Atara R, Rathi S. To evaluate and compare the effect of different post surface treatments on the tensile bond strength between fiber post and composite resin. J Int Oral Health. 2013; 5(5): 27-32.

4. Sharma A, Samadi F, Jaiswal JN, Saha S. A comparative evaluation of effect of different solvents on the shear bond strength of glass reinforced post to core material. Int J Clin Pediatr Dent. 2014; 7(3): 192-6.

5. Cecchin D, Farina AP, Pino R, Ratto R. Acid etching and surface coating of glass-fiber posts: Bond strength and interface analysis. Braz Dent J. 2016; 27(2): 228-33.

6. Saker S, El-Kholany N, El-Wassefy N. Effect of different surface treatments on push-out bond strength of glass fiber posts to resin composite core material. J Dent App. 2015; 2(6): 246-50.

7. Coelho A, Rezende A, Gomes P, et al. Critical analysis of fiber post surface treatment: A review Biosci J. 2014; 30(3): 914-24.

8. Mosharraf R, Ranjbarian P. Effect of post surface conditioning before silanization on bond strength between fiber post and resin cement. J Adv Prosthodont. 2013; 5(2): 126-32.

9. Zago L, Ribeiro F, Guimarães C, et al. Surface treatment of glass fiber and carbon fiber posts: SEM characterization. Microsc Res Tech. 2011; 74: 1088-1092. Doi: http://dx.doi.org/10.1002/jemt.20999

10. Scotti R, Ferrari M. Pernos de fibra. Bases teóricas y aplicaciones clínicas. Segunda Edición. Barcelona: Masson; 2004.

11. De Sousa M, Cavalcanti E, Vinícius P, Faria-e-Silva AL, Soares CJ, Marcondes LR. Fiber post etching with hydrogen peroxide: Effect of concentration and application time. JOE. 2011; 37(3): 398-402.

12. Sahafi A, Peutzfeldt A. Durability of the bond between resin composite cores and prefabricated posts. Acta Odontol Scand. 2009; 67(5): 271-6.

13. Bitter K, Neumann K, Kielbassa AM. Effect of salinization on bond strenght of fiber posts to various resin cements. Quintessence Int. 2007; 38(2): 121-28.

14. Mukund C, Meena A, Chitre V. Surface conditioning treatments for improving adhesion of fiber posts. Journal of Orofacial Research. 2012; 2(1): 37-41.

15. Sahafi A, Peutzfeldt A. Durability of the bond between resin composite cores and prefabricated posts. Acta Odontol Scand. 2009; 67(5): 271-6.

16. Mukund C, Meena A, Chitre V. Surface conditioning treatments for improving adhesion of fiber posts. Journal of Orofacial Research. 2012; 2(1): 37-41.

17. Mazzitelli C, Ferrari M, Toledano M, Osorio E, Monticelli F, Osorio R. Surface roughness analysis of fiber post conditioning processes. J Dent Res. 2008; 87(2): 186-90.

18. Hidalgo R. Reacción de la dentina a los sistemas adhesivos resinosos: aspectos biológicos relacionados y biodegradación de la capa híbrida. Rev Estomatol Herediana. 2008; 18(1): 50-64.

19. Herrera D, Kose C, Villa F, Stanislawczuk R, Reis A, Dourado A. Clorhexidina como alternativa para maximizar la longevidad de restauraciones adhesivas. Rev Estomatol Herediana. 2010; 20(7): 78-84.

Recibido: 12-04-2017

Aceptado: 05-07-2017 